\section{Research Square}

\title{
Etoricoxib decreases mouse subchondral bone mass and biomechanical properties in early osteoarthritis
}

Bo Liu

Institute of Orthopaedics, Soochow University

Chen-Chen Ji

Institute of Orthopaedics, Soochow University

Yi-Jie Shao

Institute of Orthopaedics, Soochow University

Ting Liang

Institute of Orthopaedics, Soochow University

Jia-Heng He

Institute of Orthopaedics, Soochow University

Hua-Ye Jiang

Institute of Orthopaedics, Soochow University

Guang-Dong Chen

Institute of Orthopaedics, Soochow University

Zong-Ping Luo ( $\nabla$ zongping_luo@yahoo.com )

Orthopedic Institute https://orcid.org/0000-0002-0617-2597

\section{Research article}

Keywords: Etoricoxib, COX-2, osteoarthritis, microstructure, subchondral bone

Posted Date: January 7th, 2020

DOI: https://doi.org/10.21203/rs.2.20150/v1

License: (1) (1) This work is licensed under a Creative Commons Attribution 4.0 International License. Read Full License 


\section{Abstract}

\section{Background}

Etoricoxib, a selective Cyclooxygenase-2 (COX-2) inhibitor, is commonly used in osteoarthritis (OA) for pain relief. The purpose of our study was to investigate the effects of Etoricoxib on mouse subchondral bone in early $\mathrm{OA}$.

\section{Methods}

OA was induced via destabilization of the medial meniscus (DMM) in C57BL/6J mice. After surgery, the mice were randomly and equally divided into five groups: a sham-operated control group (Sham group), an osteoarthritis (OA) group (DMM group), an OA treated with Etoricoxib 5mg/kg (DMM+E5) group, an OA treated with Etoricoxib $10 \mathrm{mg} / \mathrm{kg}$ (DMM+E10) group, and an OA treated with Etoricoxib $20 \mathrm{mg} / \mathrm{kg}$ (DMM+E20) group. Mice in the Sham group and DMM group were injected with a similar dose of vehicle (40\% ethyl alcohol-saline solution). Four weeks after treatment, mice were euthanized. Micro computed tomography (Mirco-CT) analysis, Safranin O-Fast Green staining, hematoxylin and eosin (HE) staining were performed to evaluate morphological and structural changes. In addition, atomic force microscopy (AFM) analysis was performed to evaluate changes in the elastic modulus. Furthermore, changes in microstructure were detected by scanning electron microscopy (SEM).

\section{Results}

Etoricoxib inhibited osteophyte formation in the subchondral bone. However, it also reduced the bone volume fraction (BV/TV), lowered trabecular thickness (Tb.Th), and more microfractures and pores were observed in the subchondral bone. Moreover, Etoricoxib reduced the elastic modulus of subchondral bone. Furthermore, exposure to Etoricoxib further increased the empty/total osteocyte ratio of the subchondral bone. In cartilage and synovium, Etoricoxib did not significantly change the Osteoarthritis Research Society International (OARSI) score, the modified Mankin score, and the synovialitis-score versus the DMM group.

\section{Conclusion}

Our results demonstrate that although Etoricoxib can relieve the pain induced by $\mathrm{OA}$, it also has adverse effects on subchondral bone in early OA.

\section{Background}

Osteoarthritis (OA), the most common type of arthritis, is a chronic degenerative joint disease that is characterized by articular cartilage degeneration, subchondral osteosclerosis, osteophyte formation, and synovial inflammation, which has a high morbidity in the geriatric population [1, 2]. Previous studies on OA mainly focused on articular cartilage degeneration. However, the differences between the results of preclinical research and clinical medication of OA have shown that targeting articular cartilage alone is 
not enough to prevent the progress of OA [3]. Not only cartilage, but also subchondral bone plays an important role in the pathogenesis of OA. Therefore, OA has been considered an organ disease that affects the whole joint [4-6]. Some studies have revealed that articular cartilage and subchondral bone make up a functional joint unit, which plays a complementary role in the weight bearing capacity of the joint [7].

Pain is the most typical clinical manifestation of OA patients, and it is the main cause of disability and medical medication in patients with knee joint $\mathrm{OA}[8,9]$. In addition, joint pain has adverse effects on the quality of life of patients [10]. Therefore, treatments for OA have focused on relieving pain symptoms [2]. At present, oral non-steroidal anti-inflammatory drugs (NSAIDs), especially Cyclooxygenase-2 (COX-2) inhibitors are the most commonly analgesics used for treatment of OA and are often recommended in the guidelines for clinical practice of OA [11-14]. In the clinic, Etoricoxib, a selective COX-2 inhibitor, is commonly used in OA for pain relief. The anti-inflammatory and analgesic effects of Etoricoxib are mainly due to its capacity to inhibit cyclooxygenase (COX), thereby impairing the production of prostaglandin E2 (PGE2), which are vital mediators of pain and inflammatory responses [15-18]. In addition to its role in pain and inflammatory responses, recent studies have shown that PGE2 plays multiple roles in bone metabolism [15]. The stimulatory or inhibitory effects of PGE2 on bone metabolism depend on different structure-activity relations and signaling pathways $[19,20]$. In a previous study, it was revealed that PGE2 secreted by osteoblastic cells activated PGE2 receptor 4 (EP4) in sensory nerves to regulate bone formation by inhibiting sympathetic activity through the central nervous system [21].

In recent years, many studies have demonstrated the effects of COX-2 inhibitors on bone, however, the effects of COX-2 inhibitors (such as Etoricoxib) on subchondral bone in OA are still unclear, especially the role of COX-2 inhibitors on its microstructure [11, 22-24]. The microscopic biomechanical environment of knee subchondral bone is very important for the repair/degeneration of subchondral bone cells [25].

In this study, we hypothesized that Etoricoxib is involved in changes of the microstructure and biomechanical properties of knee subchondral bone during treatment of early knee OA. To test this hypothesis, we studied mice with OA after intraperitoneal injection of three concentrations of Etoricoxib. The results of this study may be of great significance to determine the effects of Etoricoxib on microscopic biomechanical environment of subchondral bone in patients with early knee OA, and may play an important guiding role on the treatment of OA.

\section{Methods}

\section{Experimental animals}

A total of 75 male C57BL/6J mice (8 weeks, 20 $2 \mathrm{~g}$ ) were purchased from JOINN Laboratories (Suzhou), Inc. (License No. SCXK(Su)2018-0006, Suzhou, China). Following arrival at the facility, mice were allowed at least 1 week to acclimatize [26]. Experimental animals (3-5 mice/cage) were housed in a room at $23 \pm$ $2^{\circ} \mathrm{C}$, with $55 \pm 10 \%$ humidity and lighting was maintained at a 12-hours-on-12-hours-off schedule (light on from 7:00 a.m. to 19:00 p.m.). Food and water were provided ad libitum. Maintenance, use, and treatment 
of all animals were in accordance with accepted standards of the Ethics Committee of Soochow University (ECSU-2019000158).

\section{Destabilization of the medial meniscus (DMM) surgery}

After 1 week of adaptive feeding, the medial meniscal ligament (MMLT) in the right knee joint was severed to establish an OA model as described previously [27, 28]. In brief, after isoflurane inhalation anesthesia in mice, a medial incision of the right knee joint was created to slightly shift the extensor muscle of the knee joint without transecting the patellar ligament and expose the right knee joint. The MMLT was cut, so that the medial meniscus could be moved to the medial side. After repositioning the knee extensor, the medial incision was sutured and the skin closed. For mice that underwent sham surgery, a similar surgical approach was used, however, the knee joint was operated without MMTL transection. After surgery, mice were had access to food and tap water ad libitum.

\section{Grouping, drug medications, and treatment protocols}

Two days after DMM surgery, mice were randomly and equally divided into five groups ( $n=15$ in each group), including a sham-operated control group (Sham group), an osteoarthritis (OA) group (DMM group), an OA treated with Etoricoxib $5 \mathrm{mg} / \mathrm{kg}$ (DMM+E5) group, an OA treated with Etoricoxib $10 \mathrm{mg} / \mathrm{kg}$ (DMM+E10) group, and an OA treated with Etoricoxib 20mg/kg (DMM+E20) group.

Etoricoxib was purchased from Jiangsu argon krypton xenon material technology co. LTD (Suzhou, China). Etoricoxib and the vehicle ( $40 \%$ ethyl alcohol-saline solution) were freshly prepared before each injection, Etoricoxib was dissolved in the vehicle solution and placed in a warm bath until completely dissolved. According to the grouping scheme mentioned above, mice were injected intraperitoneally, 3 times a week for 4 weeks with an injection dose of $0.1 \mathrm{~mL}$ per mouse. Mice in the Sham group and DMM group were injected with a similar dose of vehicle (Figure 1).

\section{Micro computed tomography measurements}

Four weeks after a 2-days recovery period, mice were euthanized by continuous $\mathrm{CO}_{2}$ inhalation, the right knee joints were harvested, and fixed in $10 \%$ neutral formalin. After fixation for 48 hours, the specimens were transferred to $70 \%$ ethanol for high-resolution micro computed tomography (Micro-CT) (SkyScan1176, Aartselaar, Belgium) [29]. The scanner was set at a gamma-ray voltage of 50KV and a current of $200 \mathrm{uA}$, the filter was $0.5 \mathrm{~mm} \mathrm{Al}$, and a resolution of $9 \mu \mathrm{m}$ per pixel was used. The standardized parameters and thresholds of grey values for all samples were $0-0.075$. The NRecon software and NReconServer software were used for the reconstruction of two-dimensional images, and Dataview software was applied to adjust the XYZ axes. mages were analyzed using the CTan software, and sagittal and coronal images of the tibial subchondral bone were applied to evaluate changes. The subchondral bone of the medial tibial plateau with 15 consecutive layers in the regions of interest (ROI) in the recombinant images of mice was selected for three-dimensional reconstruction and quantitative analysis. 
Specific indicators included bone volume fraction (BV/TV) and trabecular thickness (Tb.Th). Threedimensional images were acquired by Mimics software.

\section{Atomic force microscopy analysis and nano-mechanical testing}

Fresh knee joints were dehydrated in $20 \%$ and $30 \%$ sucrose solution for 12 hours, respectively. After dehydration, knee joints were embedded with optimal cutting temperature compound and cut into 20-30 $\mu \mathrm{m}$ thick sections by a section microtome (CM3050S, Leica, Nussloch, Germany). From each group, four slides were selected, and five areas were taken from each slide, which were scanned by atomic force microscopy (AFM) (Dimension ICON, Bruker, USA). AFM tests were carried out at room temperature (RT). First, the force constant $\mathrm{k}(40 \mathrm{~N} / \mathrm{m})$ of the probe (Brooke, Germany) and the curvature radius $\mathrm{R}(5 \mathrm{~nm})$ of the tip was calibrated. Then, we performed tests in the ROI of the subchondral bone, obtained the mechanical curve, and calculated the elastic modulus by using the formula (1). In this study, the Hertz model, which is the most commonly used in the AFM test of biological tissue, is used to calculate the compression modulus of the elastic modulus. The formula (1) is as follows:

$$
\mathrm{F}=\frac{4}{3} \frac{E}{\left(1-v^{2}\right)} \sqrt{R} \delta^{3 / 2}
$$

$F, E, v, R$ and $\delta$ represent pressure, Young's modulus, Poisson's ratio, indentation radius, and indentation depth, respectively.

\section{Scanning electron microscopy analysis}

After excess tissue was removed from fresh knee joints, knee joints were digested with a mixed enzyme solution comprised of type I and type II collagenase (4\% type I collagenase mixed with $4 \%$ type II collagenase). The subchondral bone was obtained by placing the knee joints in an incubator at $37^{\circ} \mathrm{C}$ and by changing the digestive solution every day until the cartilage and surrounding soft tissues were completely digested. After digesting, the specimens were washed with phosphate buffered solution, then fixed with $4 \%$ glutaraldehyde for 2.5 hours at RT, rinsed in phosphate buffered solution, and dehydrated in graded ethanol series. After the residual moisture was further removed by the critical point dryer, the specimens were fixed on a metal platform through conductive glue, then sprayed with gold by ion spatter to enhance its conductivity. Finally, specimens were observed by scanning electron microscopy (SEM) (FEl Quanta 250, Hillsboro, USA).

\section{Histomorphometry analysis}

After Mirco-CT imaging, samples were decalcified in $10 \%$ ethylenediamine tetraacetic acid (EDTA) $(\mathrm{pH}=7.4)$ on a shaker at RT for 14 days. Following decalcification, and trimming away excess soft tissues, knee joints were dehydrated in graded ethanol series. Then, samples were transferred to N-butyl alcohol for 8 hours and infiltration with paraffin was performed for 7 hours. Finally, paraffin-embedded tissues were cut into 6- $\mu \mathrm{m}$-thick sections using a rotary microtome. Hematoxylin-eosin (HE) staining was adopted to evaluate pathological changes of cartilage of medial tibial and subchondral bone of medial tibial and 
synovial tissues, and cartilage, subchondral bone, and synovium were evaluated by the modified Mankin score, the empty/total osteocyte ratio, and the synovialitis-score, respectively [30-33]. Safranin 0-Fast Green staining was performed on sagittal sections to determine changes in proteoglycans, and cartilage destruction was scored using the Osteoarthritis Research Society International (OARSI) OA cartilage histopathology assessment system as described [34].

\section{Statistical analysis}

Statistical analysis was performed using SPSS 23.0 (SPSS Inc., Chicago, IL, USA) and GraphPad prism 7.0 (GraphPad Software, La Jolla, CA, USA). Normal distribution and homogeneity of variance of data were evaluated by Shapiro-Wilk test and Levene's test, respectively. Data were presented as the mean \pm standard deviation (SD). Significant differences between study groups were obtained using a one-way analysis of variance (ANOVA) with Tukey's post-hoc test. Statistical significance was set at $p[0.05$.

\section{Results}

\section{In vivo Micro-CT revealed DMM-operated mice exposure to Etoricoxib further reduced the bone mass of subchondral bone}

Micro-CT was used to quantitatively evaluate the subchondral bone of the medial tibial plateau. As shown in Figure 2A, both sagittal and coronal images showed an increase in bone mass of the subchondral bone of the medial tibial plateau in the DMM group when compared with the Sham group, showing a state of "osteosclerosis". However, in the treatment groups, the bone mass of the subchondral bone of the medial tibial plateau was significantly lower compared to that in the DMM group, and even lower compared to that in the Sham group. In addition, the subchondral bone of the treatment groups showed an increase in the number of cysts.

As shown in Figure 2C and Figure 2D, four weeks after a 2-day recovery, BV/TV increased significantly in the DMM group when compared with the Sham group (P凶0.001). In addition, when compared with the DMM group, BV/TV significantly decreased after intraperitoneal injection of Etoricoxib ( $p<0.001$ for $\mathrm{DMM}+\mathrm{E} 5, \mathrm{DMM}+\mathrm{E} 10$ and $\mathrm{DMM}+\mathrm{E} 20)$, which was significantly decreased when compared with the Sham group (Pख0.05 for all dosages). Furthermore, BV/TV in DMM+E20 group was lower than that in DMM+E5 group (Pख0.01). In the DMM group, the Tb.Th was significantly higher compared to that in the Sham group (Pख0.001), meanwhile, it also higer than that in treatment groups (P区0.001 for all dosages). Tb.Th was decreased after treatment with Etoricoxib and in DMM+E20 group it was significantly lower compared to that in the Sham group (Pख0.05). Moreover, Tb.Th in DMM+E20 group was lower than that in $\mathrm{DMM}+\mathrm{E} 5$ group (Pख0.05). Overall, at the early stage of $\mathrm{OA}$, the subchondral bone of the medial tibial plateau showed increased bone mass, while the subchondral bone mass decreased and was lower compared to that in the Sham group after treatment with Etrecoxib.

\section{AFM evaluation of subchondral bone showed that Etrecoxib reduced the elastic modulus of DMM- induced subchondral bone}


The elastic modulus of subchondral bone obtained from the knee joints of mice can be measured at micrometre and nanometre scales using AFM. As shown in Figure 3, the elastic modulus of subchondral bone decreased by $(1.048 \pm 0.236 \mathrm{GPa})$ in DMM group $(6.968 \pm 0.147 \mathrm{GPa})$ when compared with the Sham group $(8.016 \pm 0.185 \mathrm{GPa})(\mathrm{P}<0.001)$. However, when compared with the Sham group, the elastic modulus of subchondral bone in DMM+E5 group (6.378 $\pm 0.150 \mathrm{GPa}), \mathrm{DMM}+\mathrm{E} 10$ group $(6.198 \pm 0.177$ $\mathrm{GPa})$, and $\mathrm{DMM}+\mathrm{E} 20$ group $(6.127 \pm 0.143 \mathrm{GPa})$ decreased by $(1.638 \pm 0.238 \mathrm{GPa})(\mathrm{P}<0.001),(1.819$ $\pm 0.256 \mathrm{GPa})(\mathrm{P}<0.001)$ and $(1.889 \pm 0.234 \mathrm{GPa})(\mathrm{P}<0.001)$, respectively. Moreover, when compared with the $\mathrm{DMM}$ group, the elastic modulus of the $\mathrm{DMM}+\mathrm{E} 10$ group and $\mathrm{DMM}+\mathrm{E} 20$ group decreased by $(0.771 \pm 0.230 \mathrm{GPa})(\mathrm{P}<0.01)$ and $(0.841 \pm 0.205 \mathrm{GPa})(\mathrm{P}<0.01)$, respectively.

\section{SEM showed that after Etoricoxib treatment, there were more microfractures and pore density in subchondral bone, while osteophyte formation decreased}

SEM was used to evaluate the subchondral bone in each group (Figure 4A and Figure 4B). In the Sham group, the surface of subchondral bone was smooth, there were a few microfracture lines, and no osteophyte formation was observed in the subchondral bone. In the DMM group, there were also a few microfracture lines, and osteophyte was observed at the edge of the subchondral bone, hyperosteogeny can be seen in the load-bearing sites. In the treatment groups, no obvious osteophyte formation was observed in the subchondral bone. However, the microfracture lines of the subchondral bone in the treatment groups were significantly increased compare to that in the DMM group, and the microfracture lines were longer and deeper.

To further understand the subchondral bone, we statistically analyzed the pore density within the ROI of the subchondral bone (Figure 4C). Four weeks after a 2-day recovery period, the pore density in the DMM group was higher compared to that in the Sham group ( $\mathrm{p} \otimes 0.05$ ). When compared with the Sham group, the pore density significantly increased after treatment with Etoricoxib (P囚0.001 for all dosages), and in the DMM+E20 group, the pore density was significantly higher compared to that in the DMM group (PV 0.001). Furthermore, we noticed that the pore density in the DMM+E20 group was significantly higher compared to that in the DMM+E5 group and the DMM+E10 group (Pख0.05 for DMM+E5 and DMM+E10).

\section{HE staining of subchondral bone showed that exposure to Etoricoxib further increased the empty/total osteocyte ratio of subchondral bone}

To confirm the effect of Etoricoxib on subchondral bone, the empty osteocyte density $\left(/ \mathrm{mm}^{2}\right)$ and total osteocyte density $\left(/ \mathrm{mm}^{2}\right)$ in the subchondral bone were counted, and the empty/total osteocyte ratio was calculated. As shown in Figure 5D, the empty/total osteocyte ratio in the DMM group $(0.040 \pm 0.005)$ was higher compared to that in the Sham group $(0.011 \pm 0.004)(P \llbracket 0.05)$. Moreover, the ratio was increased after treatment with Etoricoxib, and in DMM+E20 group the ratio was significantly higher compared to that in DMM group (P凶0.01). Moreover, the empty/total osteocyte ratio in the DMM+E20 group was significantly higher compared to that in the DMM+E5 group and the DMM+E10 group (Pख0.05 for $\mathrm{DMM}+\mathrm{E} 5$ and $\mathrm{DMM}+\mathrm{E} 10)$. 


\section{Histologic evaluation of cartilage integrity and synovial inflammation showed that Etrecoxib has no obvious anti-inflammatory effects and does not inhibit cartilage degeneration}

To evaluate the degeneration of the emedial tibial cartilage of knee joint in each group, Safranin 0-Fast Green staining, HE staining were performed. As shown in Figure 6A and Figure 6B, four weeks after a 2day recovery, the Sham group showed a surface that was intact, the morphology of cartilage was integrated, and the loss of proteoglycans in articular cartilage was not significant. In the DMM group, proteoglycans had decreased in the superficial part of articular cartilage, and the surface of cartilage was rough and discontinuous. In the treatment groups, we found loss of proteoglycans and an uneven cartilage surface.

In the medial tibia plateau, the most affected region, the OARSI score (Figure $6 \mathrm{C}$ ) and the modified Mankin score (Figure 6D) were significantly higher in the DMM group versus that in the Sham group (P区 0.001). However, treatment with Etrecoxib had no significant effect on DMM-induced cartilage degeneration when compared with DMM (Pख0.05 for all dosages). In addition, the synovialitis-score (Figure 7) was significantly increased in OA knees (Pख0.001). However, when compared with the DMM group, the synovialitis-scores did not show an obvious change after intraperitoneal injection of Etoricoxib (p囚0.05 for DMM+E5, DMM+E10 and DMM+E20).

\section{Discussion}

In the present study, the effects of intraperitoneally administered Etoricoxib were evaluated in a mouse OA model. The results suggested that although Etoricoxib can reduce the sclerosis of subchondral bone in the medial tibial plateau, excessive loss of subchondral bone mass may occur, which has adverse effects on the joint. Specifically, Etoricoxib leads to a decrease in subchondral bone mass and elastic modulus, and can also lead to the formation of microfractures in the subchondral bone. Furthermore, Etoricoxib can inhibit osteophyte formation of the subchondral bone, however, no significant improvement was observed in articular cartilage destruction and synovial inflammation at the early stage of $O A$.

Multiple studies have shown that subchondral bone plays a critical role in the occurrence and development of OA $[35,36]$. Remodeling of subchondral bone is an important part of the progression of OA [37, 38]. Nakasa et al. [39] showed that subchondral sclerosis of the tibia could be observed 7 days after DMM, which was consistent with the findings of our study. However, after 4 weeks of treatment with Etoricoxib, significant bone mass loss was observed in the subchondral bone of the medial tibial plateau, which was below the baseline level. Previously, many studies focused on the effects of COX-2 inhibitors on bone. Goodman et al. [24] showed that COX-2 selective NSAIDs inhibited bone formation and bone growth. In many animal experiments, continuous use of NSAIDs and COX-2 inhibitors showed to inhibit bone healing [22]. This was in line with our findings. Moreover, in several studies, it was shown that COX2 inhibitors can inhibit angiogenesis and interfere with osteogenesis and osteoclast function, thereby inhibiting bone repair and formation. In an inflammatory state, high concentrations of the COX-2 inhibitor 
can reduce the content of alkaline phosphatase and calcium in bone marrow mesenchymal stem cells [40]. In a rat fracture model, short-term and long-term use of COX-2 inhibitors significantly interfered with bone adhesion and fracture healing [41]. In healthy Sprague Dawley rats, long-term use of COX-2 inhibitors stimulated bone resorption and reduced bone mass and mechanical properties of the femur [42]. In mouse studies, COX-2 inhibitors inhibited the number of bone trabeculae and calli [43]. AFM can earlier detect changes in subchondral bone structure and biomechanical properties of OA in mice, and clearly measure these changes at the micrometre and nanometre level [44]. We observed that the loss of subchondral bone mass was accompanied by the decrease of subchondral bone elastic modulus, especially in mice that were treated with Etoricoxib. The decrease in elastic modulus refers to the decrease in elastic dispersion ability, which can cause joint damage due to high frequency loads [45]. In addition, the SEM results confirmed this view, and we demonstrated that there were more microfractures and pores in the subchondral bone in the treatment groups. At the histological level, we observed that 4 weeks after the injection of Etoricoxib, the total osteocyte density decreased and the empty total osteocyte ratio increased in subchondral bone, indicating that Etoricoxib had a destructive effect on subchondral osteocytes.

The use of Etoricoxib in the early stage of knee OA relieved the symptoms of pain, however, the process of subchondral bone remodeling was disturbed, bone formation and bone destruction were seriously out of balance, and bone resorption was significantly increased. Therefore, the increase of subchondral bone loss further reduced the mechanical support capacity and the ability to absorb pressure load of the knee joint, destroys the mechanical stability environment of the knee joint, causes osteoporotic changes in subchondral bone structure, increases the risk of fragile fracture of subchondral bone, and aggravates the degeneration of OA in the knee joint. Aisa et al. [46] suggested that both non-selective and COX-2 selective NSAIDs may increase the risk of osteoporosis and fragility fractures in patients with weakened bones during analgesic or anti-inflammatory treatment.

At the same time, four weeks after injection of Etoricoxib, no inhibitory effect on cartilage destruction was observed. Consistent with these findings, Tellegen et al. [47] showed that local delivery of celecoxib had no protective effects on cartilage histology in OA mice. Moreover, no decrease in synovitis was observed between treatment groups. We cannot exclude that the absence of inhibition of synovial inflammation could be attributed to the short medication cycle.

This study had several limitations. OA is a chronic progressive disease, therefore more time points are required to monitor the development of OA to understand the changes of cartilage, subchondral bone, and synovium. In addition, the changes in inflammatory factors should be further determined by PCR and changes at the protein level should be evaluated by Western blot analysis. Therefore, future studies are of utmost importance.

\section{Conclusion}


Etoricoxib can change the microstructure and biomechanical properties of subchondral bone, promote subchondral bone loss, reduce subchondral bone quality in early OA mice, interfere with the remodeling of knee subchondral bone in the early stage, cause osteoporotic changes in subchondral bone structure, increase the risk of fragile fracture of subchondral bone, and reduce its ability to absorb pressure load. The effects of Etoricoxib in the treatment of articular cartilage degeneration and synovitis were not significant at the early stage of $O A$.

\section{Abbreviations}

COX-2: Cyclooxygenase-2; DMM: destabilization of the medial meniscus; OA: osteoarthritis; E5: Etoricoxib 5mg/kg; E10: Etoricoxib 10mg/kg; E20: Etoricoxib 20mg/kg; Micro-CT: micro computed tomography; HE: Hematoxylin-eosin; AFM: atomic force microscopy; SEM: scanning electron microscopy; BV/TV: bone volume fraction; Tb.Th: trabecular thickness; OARSI: Osteoarthritis Research Society International; NSAIDs: non-steroidal anti-inflammatory drugs; COX: cyclooxygenase; PGE2: prostaglandin E2; EP4: PGE2 receptor 4; MMLT: medial meniscus ligament; RT: room temperature; ROI: Regions of interest; EDTA: ethylenediamine tetraacetic acid; AVNOA: one-way analysis of variance.

\section{Declarations}

\section{Ethics approval and consent to participate}

All the animal experiments performed in this work were approved by the Ethics Committee of Soochow University (ECSU-2019000158).

\section{Consent for publication}

Not applicable.

\section{Availability of data and materials}

The datasets analyzed during the current study are available from the corresponding author on reasonable request.

\section{Competing interests}

The authors have declared that no competing interest exists.

\section{Funding}

This study was supported by grants from the National Natural Science Foundation of China (31570943 and 81702146), Innovation and Entrepreneurship Program of Jiangsu Province.

\section{Authors' contributions}


Experimental Design \& Interpretation of Data : Guang-Dong Chen, Zong-Ping Luo;

Experimental Operation \& Data Analysis: Bo Liu, Chen-Chen Ji, Yi-Jie Shao, Ting Liang, Jia-Heng He, and Hua-Ye Jiang;

Manuscript Preparation: Bo Liu, Guang-Dong Chen;

Manuscript Review \& Editing: Zong-Ping Luo;

Funding Acquisition: Zong-Ping Luo

Acknowledgements

Not applicable.

\section{References}

1. Kapoor M, Martel-Pelletier J, Lajeunesse D, Pelletier JP, Fahmi H: Role of proinflammatory cytokines in the pathophysiology of osteoarthritis. Nat Rev Rheumatol 2011, 7(1):33-42.

2. Charlier E, Deroyer C, Ciregia F, Malaise O, Neuville S, Plener Z, Malaise M, de Seny D: Chondrocyte dedifferentiation and osteoarthritis (OA). Biochem Pharmacol 2019, 165:49-65.

3. Hu X, Ji X, Yang M, Fan S, Wang J, Lu M, Shi W, Mei L, Xu C, Fan X et al: Cdc42 Is Essential for Both Articular Cartilage Degeneration and Subchondral Bone Deterioration in Experimental Osteoarthritis. J Bone Miner Res 2018, 33(5):945-958.

4. Castaneda S, Roman-Blas JA, Largo R, Herrero-Beaumont G: Subchondral bone as a key target for osteoarthritis treatment. Biochem Pharmacol 2012, 83(3):315-323.

5. Loeser RF, Goldring SR, Scanzello CR, Goldring MB: Osteoarthritis: a disease of the joint as an organ. Arthritis Rheum 2012, 64(6):1697-1707.

6. Liu C, Liu C, Ren X, Si L, Shen H, Wang Q, Yao W: Quantitative evaluation of subchondral bone microarchitecture in knee osteoarthritis using 3T MRI. BMC Musculoskelet Disord 2017, 18(1):496.

7. Li G, Yin J, Gao J, Cheng TS, Pavlos NJ, Zhang C, Zheng MH: Subchondral bone in osteoarthritis: insight into risk factors and microstructural changes. Arthritis research \& therapy 2013, 15(6):223.

8. Aso K, Izumi M, Sugimura N, Okanoue Y, Ushida T, Ikeuchi M: Nociceptive phenotype alterations of dorsal root ganglia neurons innervating the subchondral bone in osteoarthritic rat knee joints. Osteoarthritis Cartilage 2016, 24(9):1596-1603.

9. Malfait AM, Schnitzer TJ: Towards a mechanism-based approach to pain management in osteoarthritis. Nat Rev Rheumatol 2013, 9(11):654-664.

10. Lane NE, Brandt K, Hawker G, Peeva E, Schreyer E, Tsuji W, Hochberg MC: OARSI-FDA initiative: defining the disease state of osteoarthritis. Osteoarthr Cartil 2011, 19(5):478-482. 
11. Goodman SB, Ma T, Mitsunaga L, Miyanishi K, Genovese MC, Smith RL: Temporal effects of a COX-2selective NSAID on bone ingrowth. J Biomed Mater Res A 2005, 72(3):279-287.

12. McAlindon TE, Bannuru RR, Sullivan MC, Arden NK, Berenbaum F, Bierma-Zeinstra SM, Hawker GA, Henrotin $\mathrm{Y}, \mathrm{Hunter} \mathrm{DJ}$, Kawaguchi $\mathrm{H}$ et al: OARSI guidelines for the non-surgical management of knee osteoarthritis. Osteoarthritis Cartilage 2014, 22(3):363-388.

13. Bannuru RR, Osani MC, Vaysbrot EE, Arden NK, Bennell K, Bierma-Zeinstra SMA, Kraus VB, Lohmander $\mathrm{LS}$, Abbott $\mathrm{JH}$, Bhandari $\mathrm{M}$ et al: OARSI guidelines for the non-surgical management of knee, hip, and polyarticular osteoarthritis. Osteoarthritis Cartilage 2019.

14. Ter Heegde F, Luiz AP, Santana-Varela S, Chessell IP, Welsh F, Wood JN, Chenu C: Noninvasive Mechanical Joint Loading as an Alternative Model for Osteoarthritic Pain. 2019, 71(7):1078-1088.

15. Blackwell KA, Raisz LG, Pilbeam CC: Prostaglandins in bone: bad cop, good cop? Trends Endocrinol Metab 2010, 21(5):294-301.

16. Cho H, Walker A, Williams J, Hasty KA: Study of osteoarthritis treatment with anti-inflammatory drugs: cyclooxygenase-2 inhibitor and steroids. Biomed Res Int 2015, 2015:595273.

17. Bley KR, Hunter JC, Eglen RM, Smith JA: The role of IP prostanoid receptors in inflammatory pain. Trends in pharmacological sciences 1998, 19(4):141-147.

18. Kawabata A: Prostaglandin E2 and pain-an update. Biological \& pharmaceutical bulletin 2011, 34(8):1170-1173.

19. Lisowska B, Kosson D, Domaracka K: Lights and shadows of NSAIDs in bone healing: the role of prostaglandins in bone metabolism. Drug Des Devel Ther 2018, 12:1753-1758.

20. Salari P, Abdollahi M: Controversial effects of non-steroidal anti-inflammatory drugs on bone: a review. Inflammation \& allergy drug targets 2009, 8(3):169-175.

21. Chen H, Hu B, Lv X, Zhu S, Zhen G, Wan M, Jain A, Gao B, Chai Y, Yang M et al: Prostaglandin E2 mediates sensory nerve regulation of bone homeostasis. Nat Commun 2019, 10(1):181.

22. Seidenberg $A B, A n Y H$ : Is there an inhibitory effect of COX-2 inhibitors on bone healing? Pharmacol Res 2004, 50(2):151-156.

23. van Esch RW, Kool MM, van As S: NSAIDs can have adverse effects on bone healing. Med Hypotheses 2013, 81(2):343-346.

24. Goodman S, Ma T, Trindade M, Ikenoue T, Matsuura I, Wong N, Fox N, Genovese M, Regula D, Smith RL: COX-2 selective NSAID decreases bone ingrowth in vivo. Journal of orthopaedic research : official publication of the Orthopaedic Research Society 2002, 20(6):1164-1169.

25. Zurriaga Carda J, Lastra ML, Antolinos-Turpin CM, Morales-Román RM, Sancho-Tello M, Perea-Ruiz S, Milián L, Fernández JM, Cortizo AM, Carda $C$ et al: A cell-free approach with a supporting biomaterial in the form of dispersed microspheres induces hyaline cartilage formation in a rabbit knee model. Journal of biomedical materials research Part B, Applied biomaterials 2019.

26. McDougall JJ, Muley MM, Philpott HT, Reid A, Krustev E: Early blockade of joint inflammation with a fatty acid amide hydrolase inhibitor decreases end-stage osteoarthritis pain and peripheral 
neuropathy in mice. Arthritis Res Ther 2017, 19(1):106.

27. Glasson SS, Blanchet TJ, Morris EA: The surgical destabilization of the medial meniscus (DMM) model of osteoarthritis in the 129/SvEv mouse. Osteoarthritis Cartilage 2007, 15(9):1061-1069.

28. lijima H, Aoyama T, Ito A, Tajino J, Nagai M, Zhang X, Yamaguchi S, Akiyama H, Kuroki H:

Destabilization of the medial meniscus leads to subchondral bone defects and site-specific cartilage degeneration in an experimental rat model. Osteoarthritis Cartilage 2014, 22(7):1036-1043.

29. Bouxsein ML, Boyd SK, Christiansen BA, Guldberg RE, Jepsen KJ, Muller R: Guidelines for assessment of bone microstructure in rodents using micro-computed tomography. J Bone Miner Res 2010, 25(7):1468-1486.

30. Zhao Z, Dai XS, Wang ZY, Bao ZQ, Guan JZ: MicroRNA-26a reduces synovial inflammation and cartilage injury in osteoarthritis of knee joints through impairing the NF-kappaB signaling pathway. Biosci Rep 2019, 39(4).

31. Shao YJ, Chen X, Chen Z, Jiang HY, Zhong DY, Wang YF, Yang HL, Saijilafu, Luo ZP: Sensory nerves protect from the progression of early stage osteoarthritis in mice. Connect Tissue Res 2019:1-11.

32. Krenn V, Morawietz L, Burmester GR, Kinne RW, Mueller-Ladner U, Muller B, Haupl T: Synovitis score: discrimination between chronic low-grade and high-grade synovitis. Histopathology 2006, 49(4):358364.

33. Naito K, Watari T, Furuhata A, Yomogida S, Sakamoto K, Kurosawa H, Kaneko K, Nagaoka I: Evaluation of the effect of glucosamine on an experimental rat osteoarthritis model. Life Sci 2010, 86(13-14):538-543.

34. Pritzker KP, Gay S, Jimenez SA, Ostergaard K, Pelletier JP, Revell PA, Salter D, van den Berg WB: Osteoarthritis cartilage histopathology: grading and staging. Osteoarthritis Cartilage 2006, 14(1):1329.

35. Madry H, van Dijk CN, Mueller-Gerbl M: The basic science of the subchondral bone. Knee Surg Sports Traumatol Arthrosc 2010, 18(4):419-433.

36. Botter SM, van Osch GJ, Clockaerts S, Waarsing JH, Weinans H, van Leeuwen JP: Osteoarthritis induction leads to early and temporal subchondral plate porosity in the tibial plateau of mice: an in vivo microfocal computed tomography study. Arthritis Rheum 2011, 63(9):2690-2699.

37. Miller RE, Scanzello CR, Malfait AM: An emerging role for Toll-like receptors at the neuroimmune interface in osteoarthritis. Seminars in immunopathology 2019.

38. Liang S, Wang ZG, Zhang ZZ, Chen K, Lv ZT, Wang YT, Cheng P, Sun K, Yang Q, Chen AM: Decreased RIPK1 expression in chondrocytes alleviates osteoarthritis via the TRIF/MyD88-RIPK1-TRAF2 negative feedback loop. Aging 2019, 11(19):8664-8680.

39. Nakasa T, Ishikawa M, Takada T, Miyaki S, Ochi M: Attenuation of cartilage degeneration by calcitonin gene-related paptide receptor antagonist via inhibition of subchondral bone sclerosis in osteoarthritis mice. Journal of orthopaedic research : official publication of the Orthopaedic Research Society 2016, 34(7):1177-1184. 
40. Yoon DS, Yoo JH, Kim YH, Paik S, Han CD, Lee JW: The effects of COX-2 inhibitor during osteogenic differentiation of bone marrow-derived human mesenchymal stem cells. Stem Cells Dev 2010, 19(10):1523-1533.

41. Zhu Y, Deng G-B, Long H-T, Li K-H, Cheng L: Effects of a selective cyclooxygenase-2 inhibitor (celecoxib) on fracture healing in rats. Indian Journal of Orthopaedics 2013, 47(4).

42. Liu Y, Cui Y, Chen Y, Gao X, Su Y, Cui L: Effects of dexamethasone, celecoxib, and methotrexate on the histology and metabolism of bone tissue in healthy Sprague Dawley rats. Clin Interv Aging 2015, 10:1245-1253.

43. Spiro AS, Beil FT, Baranowsky A, Barvencik F, Schilling AF, Nguyen K, Khadem S, Seitz S, Rueger JM, Schinke $T$ et al: BMP-7-induced ectopic bone formation and fracture healing is impaired by systemic NSAID application in C57BL/6-mice. J Orthop Res 2010, 28(6):785-791.

44. Aigner T, Schmitz N, Haag J: Nanomedicine: AFM tackles osteoarthritis. Nature nanotechnology 2009, 4(3):144-145.

45. Sadeghi $H$, Shepherd DET, Espino DM: Effect of the variation of loading frequency on surface failure of bovine articular cartilage. Osteoarthritis Cartilage 2015, 23(12):2252-2258.

46. Aisa MC, Datti A, Orlacchio A, Di Renzo GC: COX inhibitors and bone: A safer impact on osteoblasts by NO-releasing NSAIDs. Life Sci 2018, 208:10-19.

47. Tellegen AR, Rudnik-Jansen I, Pouran B, de Visser HM, Weinans HH, Thomas RE, Kik MJL, Grinwis GCM, Thies JC, Woike $N$ et al: Controlled release of celecoxib inhibits inflammation, bone cysts and osteophyte formation in a preclinical model of osteoarthritis. Drug Deliv 2018, 25(1):1438-1447.

\section{Figures}

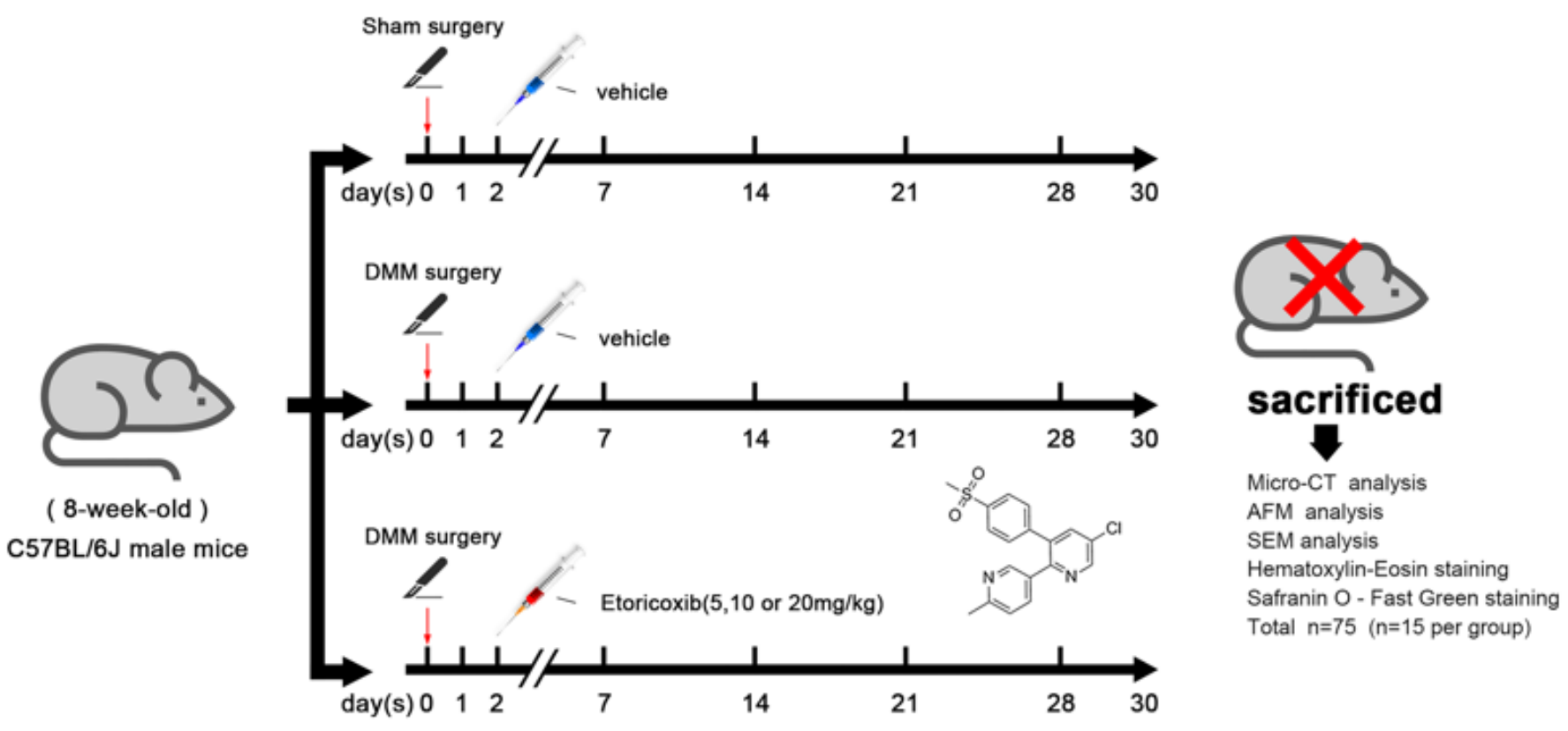


Figure 1

Schematic timeline of the experimental procedure. 8-week-old male C57BL/6J mice underwent destabilization of the medial meniscus (DMM) surgery on their right hind limbs. Mice in the Sham group underwent sham surgery on their right knee, and 2 days later, mice were either subjected to intraperitoneal injection with vehicle (Sham), vehicle (DMM), Etoricoxib $5 \mathrm{mg} / \mathrm{kg}$ (DMM+E5), Etoricoxib $10 \mathrm{mg} / \mathrm{kg}$ (DMM+E10) or Etoricoxib 20mg/kg (DMM+E20). After 4 weeks, mice were euthanatized by $\mathrm{CO} 2$ inhalation. Knee joints were collected for micro computed tomography (Micro-CT), atomic force microscopy (AFM) analysis, scanning electron microscopy (SEM) analysis, Safranin O-Fast Green staining, hematoxylin and eosin (HE) staining analysis. $n=15$ in each group.

A

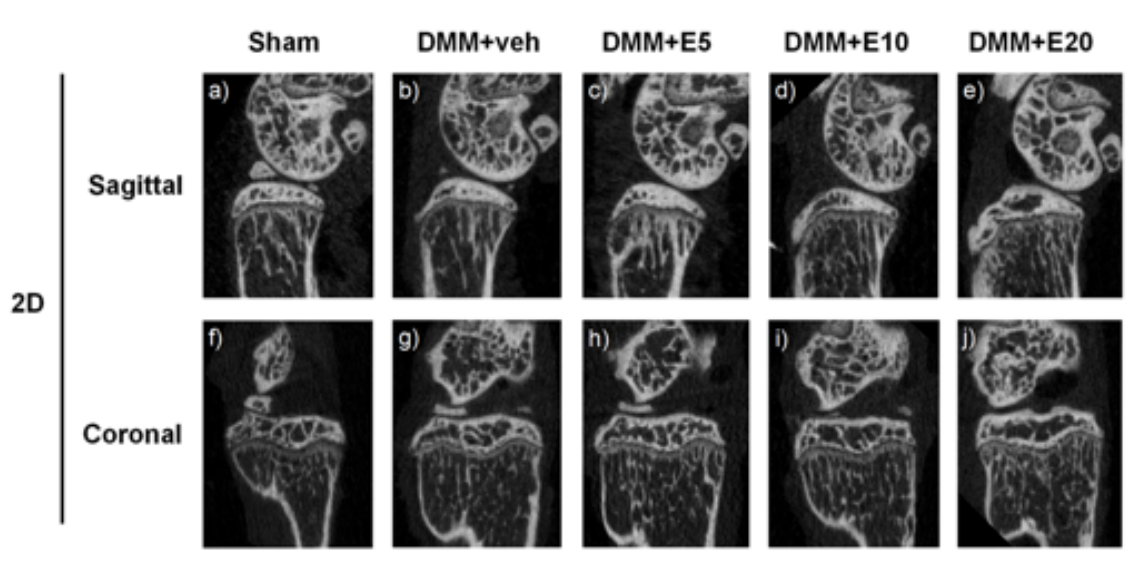

B

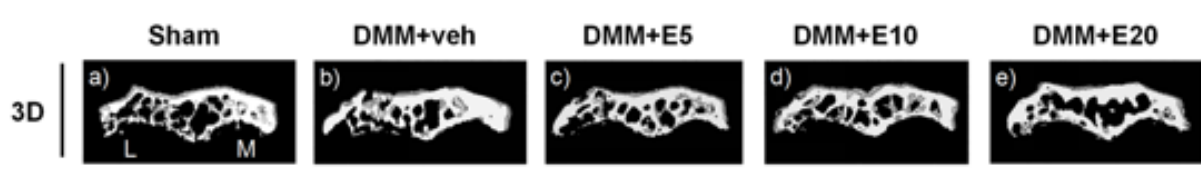

C

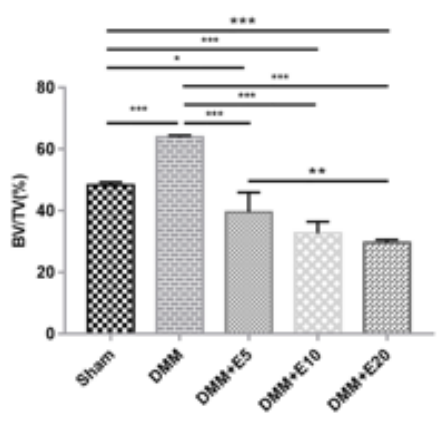

D

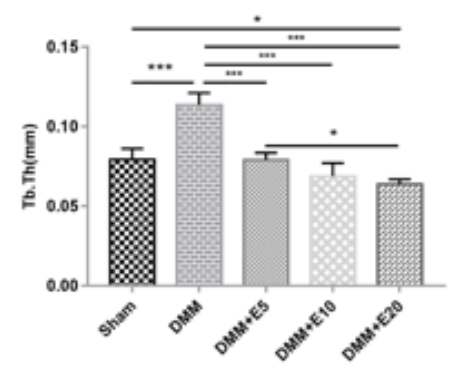

Figure 2

Micro computed tomography (Micro-CT) analysis of the medial subchondral bone of the right knee joints. (A) Representative two-dimensional Micro-CT images of medial subchondral bone compartment in both sagittal and coronal views. (B). Representative three-dimensional Micro-CT images of medial subchondral bone compartment in coronal views. (C) Quantitative analysis of bone volume fraction (BV/TV). (D) Quantitative analysis of bone trabecular thickness (Tb.Th). M: medial tibia, L: lateral tibia. * $p<0.05, * \star p<0.01, * \star * p<0.001$. 


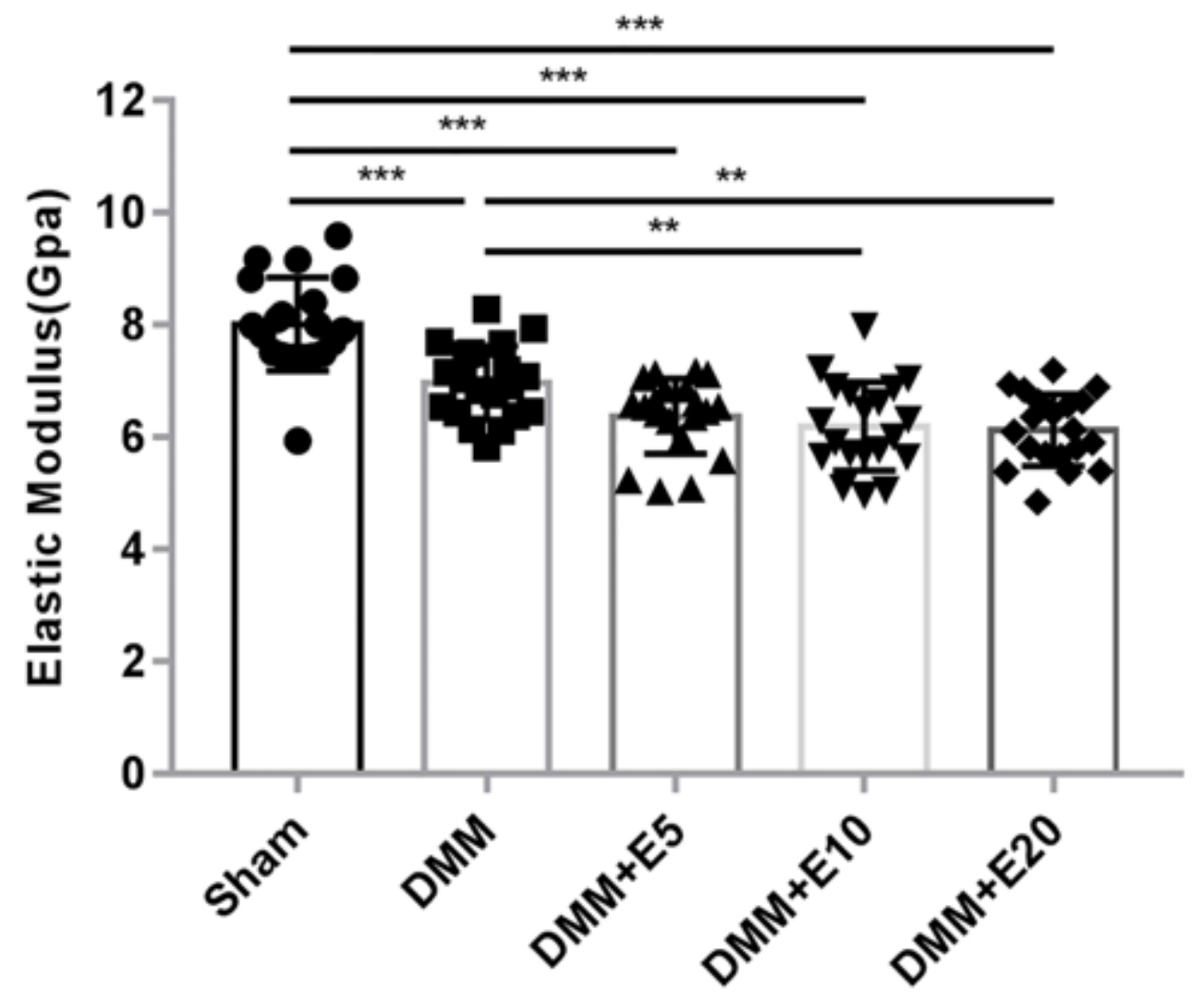

Figure 3

Atomic force microscopy (AFM) analysis of medial subchondral bone of the right knee joints.

Quantitative analysis of the elasticity modulus of medial subchondral bone. ${ }^{*} p<0.05,{ }^{*} p<0.01, * \star \star$ $\mathrm{p}<0.001$. 
A

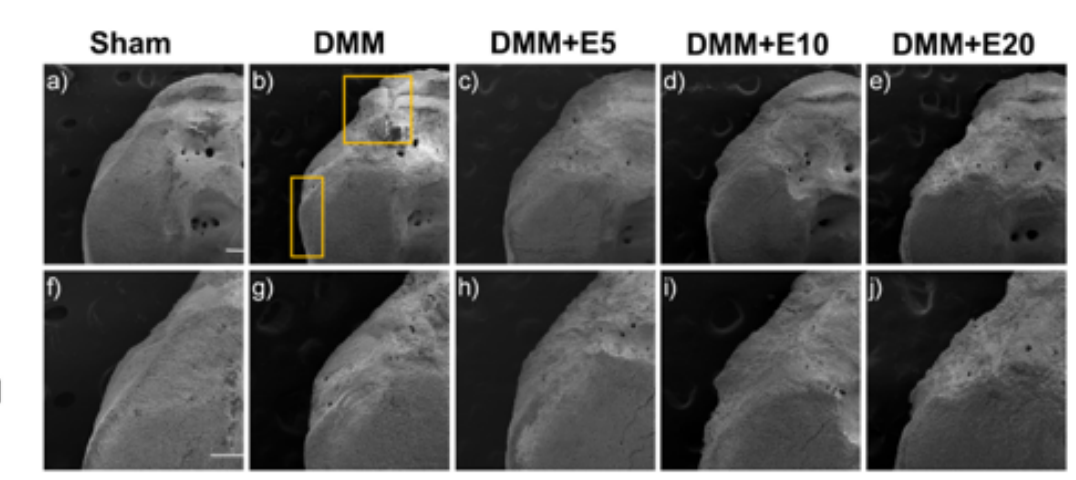

B

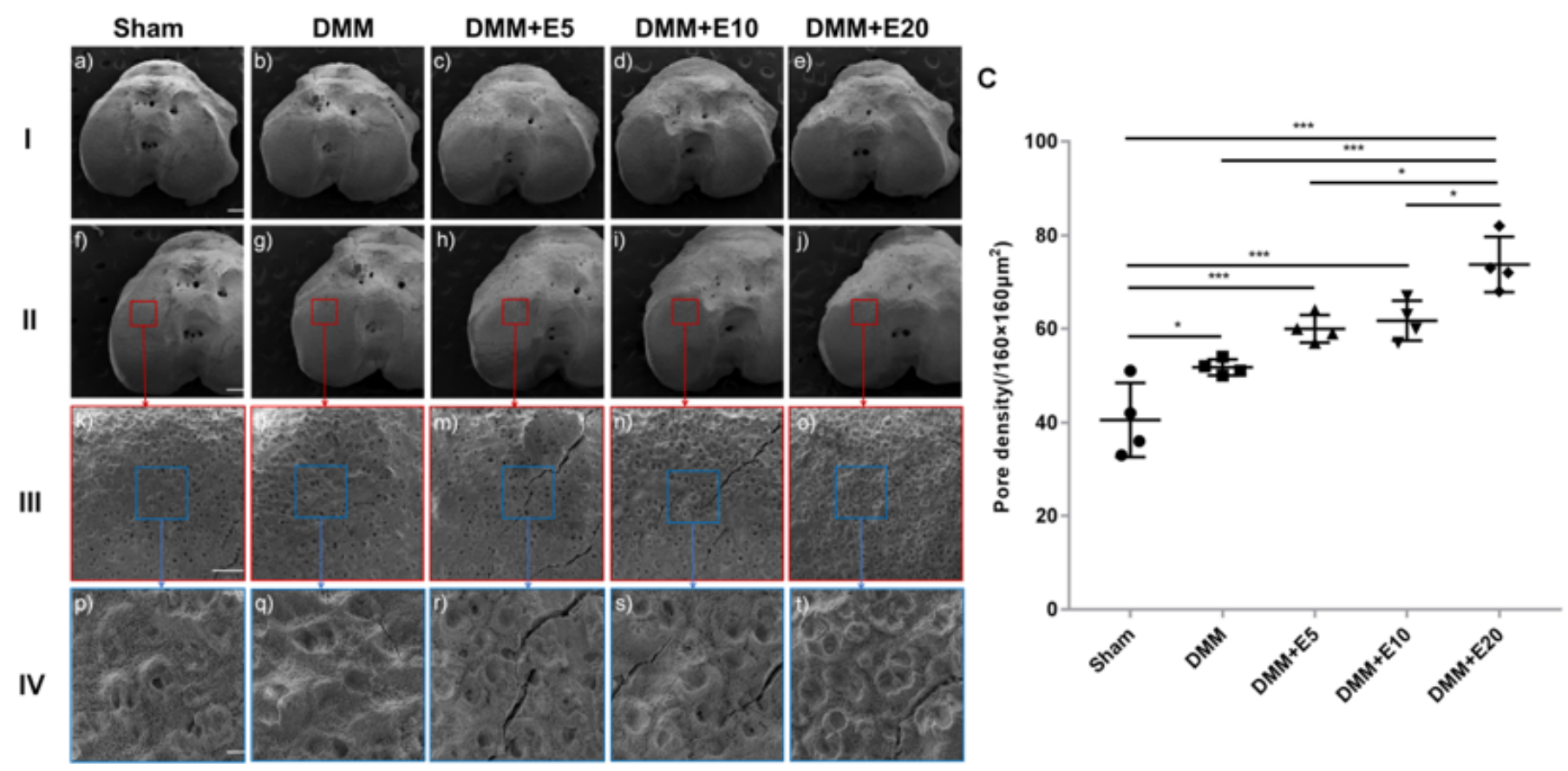

\section{Figure 4}

Scanning electron microscopy (SEM) analysis of subchondral bone. (A) Representative SEM images of the edge of the subchondral bone. The yellow solid line rectangles in row I indicate osteophytes. (B) Representative SEM images of the bearing area of the subchondral bone. The images in row III are enlarged images of the red solid line rectangle in row II. The images in row IV are enlarged images of the blue solid line rectangle in row III. (C) Quantitative analysis of the pore density $(160 \times 160 \mu \mathrm{m} 2)$ within the regions of interest $(\mathrm{ROI})$ of the subchondral bone. ${ }^{\star} p<0.05,{ }^{\star \star} p<0.01$, ${ }^{\star \star \star} p<0.001$. Scale bar of $A(I), A(I I)$, $\mathrm{B}(\mathrm{I}), \mathrm{B}(\mathrm{II}), \mathrm{B}(\mathrm{III}), \mathrm{B}(\mathrm{IV})$ was $200 \mu \mathrm{m}, 100 \mu \mathrm{m}, 300 \mu \mathrm{m}, 200 \mu \mathrm{m}, 50 \mu \mathrm{m}, 10 \mu \mathrm{m}$, respectively. 
A

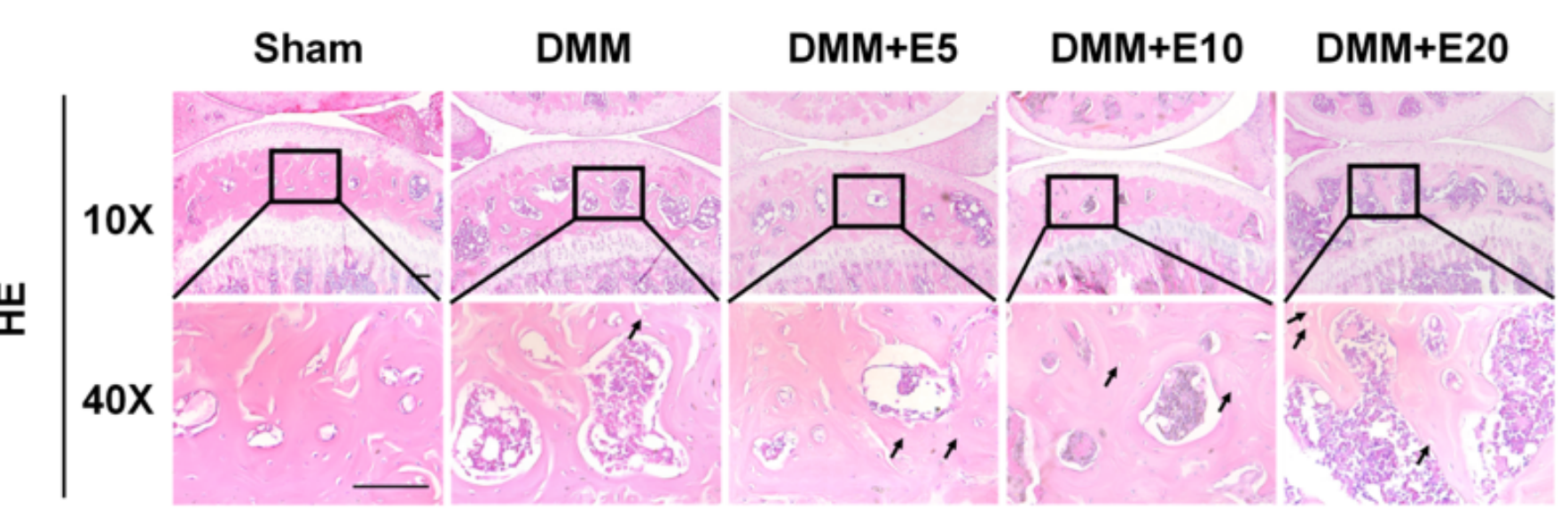

B

C

D
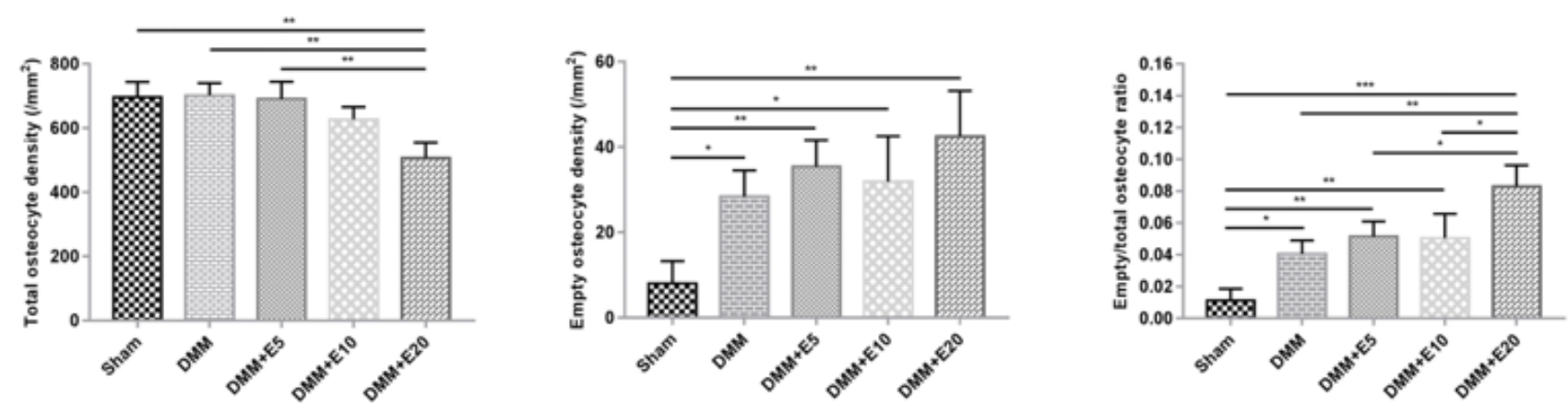

Figure 5

Effects of Etoricoxib on subchondral bone in the DMM model. (A) Hematoxylin-eosin (HE) staining of subchondral bone. Black arrows indicate empty osteocytes in subchondral bone. (B) Total osteocyte density (/mm2) of subchondral bone. (C) Empty osteocyte density (/mm2) of subchondral bone. (D) Empty/total osteocyte ratio of subchondral bone. ${ }^{\star} p<0.05,{ }^{\star \star} p<0.01,{ }^{\star \star \star} p<0.001$. Scale bar $=100 \mu \mathrm{m}$. 
A

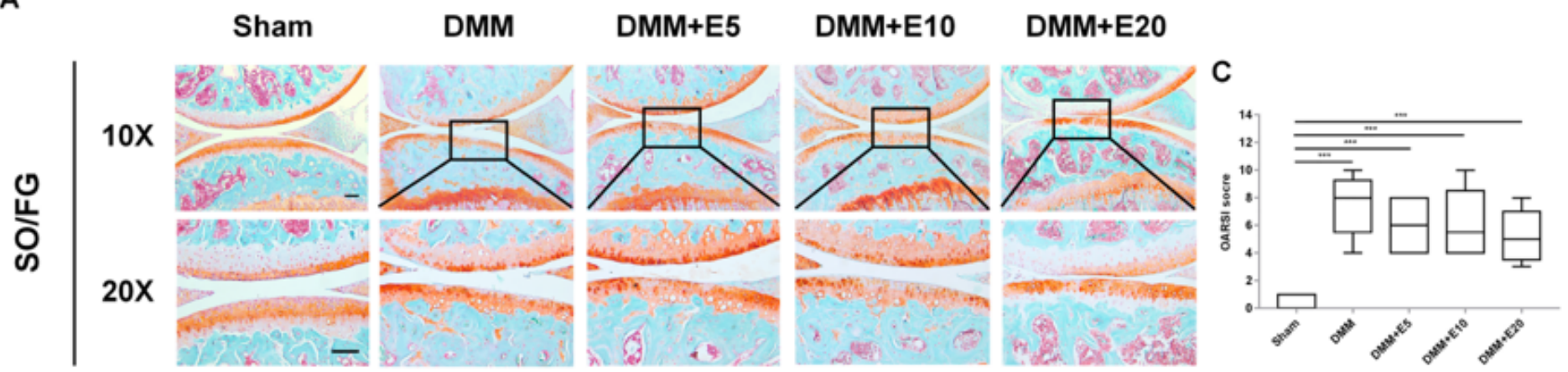

B
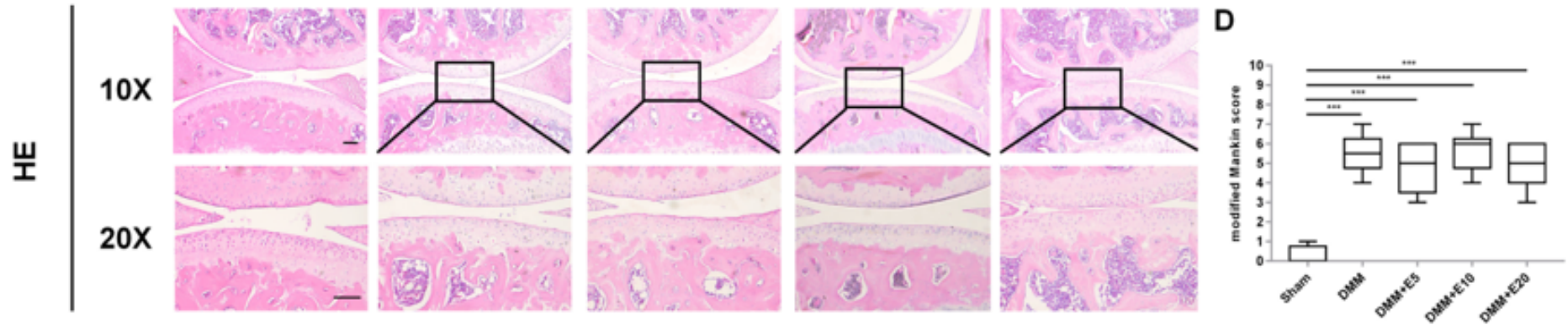

Figure 6

Effects of Etoricoxib on cartilage degeneration in the DMM model. (A) Safranin O-Fast green staining of cartilage. (B) HE staining of cartilage. (C) Osteoarthritis Research Society International (OARSI) scores for cartilage degeneration in mice. (D) modified Mankin scores for cartilage degeneration in mice. * $p<0.05$, ** $p<0.01, * * * p<0.001$. Scale bar $=100 \mu \mathrm{m}$.

A

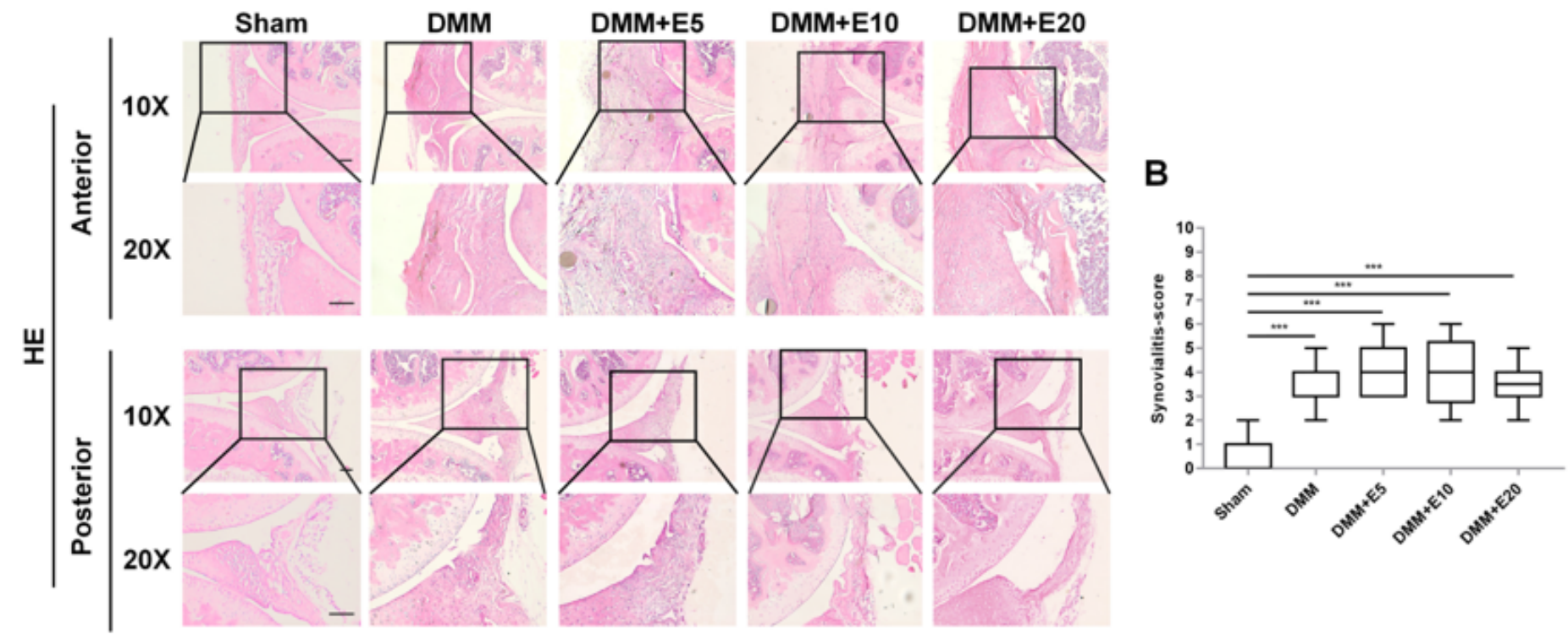

Figure 7 
Effects of Etoricoxib on synovial inflammation in the DMM model. (A) HE staining of the synovium. (B) Synovialitis-scores for synovial inflammation on mice. ${ }^{*} p<0.05, \star \star p<0.01, \star \star \star p<0.001$. Scale bar= $100 \mu \mathrm{m}$. 